\title{
Comparative studies of resonance enhancement of harmonic radiation in indium plasma using multicycle and few-cycle pulses
}

\author{
R. A. Ganeev, ${ }^{1,2}$ T. Witting, ${ }^{1}$ C. Hutchison, ${ }^{1}$ V. V. Strelkov, ${ }^{3}$ F. Frank, ${ }^{1}$ M. Castillejo, ${ }^{4}$ I. Lopez-Quintas, ${ }^{4}$ Z. Abdelrahman, ${ }^{1}$ \\ J. W. G. Tisch, ${ }^{1}$ and J. P. Marangos ${ }^{1}$ \\ ${ }^{1}$ Blackett Laboratory, Imperial College London, Prince Consort Road, London SW7 2AZ, United Kingdom \\ ${ }^{2}$ Voronezh State University, Voronezh 394006, Russia \\ ${ }^{3}$ A. M. Prokhorov General Physics Institute of RAS, Vavilova Street, 38, Moscow 119991, Russia \\ ${ }^{4}$ Instituto de Química Física Rocasolano, CSIC, Serrano 119, Madrid E-28006, Spain
}

(Received 28 May 2013; published 23 September 2013)

\begin{abstract}
Enhanced single harmonic generation is analyzed in indium laser ablation plasmas at excitation conditions of multicycle (30 fs) and few-cycle (3.5 fs) pulses. We demonstrate the strong influence of pulse duration, on the emission spectra from the indium plasma. For few-cycle pulses, the enhanced emissions do not coincide with the expected harmonic wavelengths, which is the case for multicycle pulses. We test the coherent properties of an enhanced emission around $20 \mathrm{eV}$ using polarization and double-slit interference techniques. We also characterize the dynamics of the emissions from the indium plasma by tuning the laser pulse duration. A theoretical analysis is presented to describe the indium plasma emission upon excitation by few-cycle pulses.
\end{abstract}

DOI: 10.1103/PhysRevA.88.033838

PACS number(s): 42.65.Ky

\section{INTRODUCTION}

High-order-harmonic generation (HHG) of laser radiation has long been considered a promising spectroscopic tool to retrieve structural and dynamical information regarding the nonlinear medium through analysis of the spectra, polarization states, and phase of generated harmonics [1-3]. However, the typical efficiencies of the HHG process remain below the level required for many applications of coherent extreme ultraviolet (XUV) radiation. One strategy to enhance these low efficiencies is based on exploiting the effect of resonances, which can also provide valuable information about the atomic and ionic transitions of the excited medium. The resonance peaks in the photoionization and photorecombination cross sections, including autoionizing [4], shape [5], and giant resonances [6] have long been investigated; in contrast, the studies on the role of resonances in HHG are scarce. Photorecombination, the third step in the recollision model [7-9], is the inverse process of photoionization [10] and therefore it is expected that HHG and photoionization must exhibit common resonances. This has been confirmed, not only in previous resonance effects in HHG using laser-induced transition metal plasmas [11], but also in a study of HHG from xenon gas [12].

The role of atomic resonances for increasing the laser radiation conversion efficiency has been actively discussed in the framework of perturbation theory in early studies of low-order-harmonic generation (see monograph [13] and references therein). In the case of HHG, the efficiency increase due to resonance processes came under discussion a couple of decades ago, and this approach appears to have considerable promise [14-19]. These studies comprise both the theoretical treatment and the first attempts to achieve the resonance conditions in gas HHG experiments. While theoretical estimates testify the possibility of efficient enhancement of individual harmonics and groups of harmonics, experiments in gases revealed the difficulties encountered in the observation of resonantly enhanced single harmonic [20]. Further developments have revealed that the use of laser ablation plasma media could largely facilitate the resonance enhancement of harmonics. Examination of a large group of potential solid targets has allowed the identification of some suitable materials for demonstrating this process $[21,22]$. The advantages of "plasma HHG" over "gas HHG" in resonant enhancement of harmonics became amply manifested in those studies, as the number of possible media in the former case is far greater than in the latter.

The mechanisms responsible for resonance-induced enhancement of harmonic intensity are still under debate. Some attempts in explaining the experimental observations have been reported in [23-28]. In particular, an approach [25] suggests a HHG model based on the enhancement of the generation efficiency for a particular harmonic which is resonant with a transition between the ground and an autoionizing state (AIS) of the generating ion. In that model, the third (recombination) step of the three-step scenario of HHG [7-9], is partitioned into two steps: the capture of a laser-accelerated electron into a parent ion's AIS, followed by radiative relaxation to the ground state with emission of the harmonic photon. HHG in the presence of a shape resonance was analyzed in [28] where a time-frequency analysis of the intensity and phase served to understand the mechanism of resonance-enhanced HHG. It was found that the resonance gives rise to a clear signature in the HHG spectrum irrespective of the pulse length. The time-frequency analysis of the HHG spectra supported a four-step model [25] where, for long-lived resonances, the interference occurs between the populations caused by re-collisions in different half cycles. This leads to new possibilities for XUV pulse shaping in the subfemtosecond time scale.

Such a shaping of XUV pulse has found confirmation in recently reported studies of $\mathrm{HHG}$ in laser-produced manganese plasma using sub-4-fs pulses [29]. The measured HHG spectra exhibited resonant enhancement in the spectral region around the $31^{\text {st }}$ harmonic of Ti:sapphire laser $(\sim 50 \mathrm{eV})$ where the intensity contrast relative to the adjacent harmonics exceeded one order of magnitude. This finding was in sharp contrast with the results of harmonic generation in Mn plasma plumes 
reported previously for multicycle laser pulses [11]. It was demonstrated that the application of few-cycle pulses may significantly change the pattern of resonance harmonics. The reported weak carrier envelope phase (CEP) dependence [29] might reduce the requirements for CEP stabilization of the few-cycle laser pulses. Moreover, without the gratings-induced losses, one can use thin metal filters to get almost single harmonic, which could be used in various applications where the coherent, ultrashort XUV pulses are required.

To address the issue of resonance enhancement of HHG in depth and analyze the validity of the proposed mechanism [29] for subfemtosecond XUV pulse generation, herein we present an investigation of the comparative behavior of the resonant harmonics generated in an indium plasma at the conditions of multi- (30 fs) and few-cycle (3.5 fs) pulses excitation. We demonstrate the strong influence of the pulse duration on the intensity of the resonantly enhanced harmonics generated from this plasma. Our studies show that, analogously to experiments with multicycle pulses in a tin plasma [30], the enhancement of harmonics in the case of few-cycle pulses does not require the exact coincidence of the frequencies of the plasma ion transitions and the harmonic frequencies of driving radiation. We also report, in the case of excitation by few-cycle pulses, polarization, CEP, and coherence measurements of the enhanced emissions from the indium plasma. Finally, our theoretical modeling suggests that harmonic emission should depend on the CEP of the driving pulse, while experimental observations show less influence of this parameter on the dynamics of harmonic spectra.

\section{EXPERIMENTAL ARRANGEMENTS}

We used two femtosecond lasers, which delivered few-cycle and multicycle pulses. First, a Ti:sapphire laser (Femtolasers Produktions $\mathrm{GmbH}$ ) provided pulses of 25 -fs (FWHM) duration and energies per pulse of up to $0.8 \mathrm{~mJ}$ at a repetition rate of $1 \mathrm{kHz}$. These pulses were focused into a $1-\mathrm{m}$-long differentially pumped hollow core fiber filled with neon at a pressure of 3 bar. The spectrally broadened pulses at the output of the fiber system were compressed using chirped mirrors. A pair of fused silica wedges served to fine tune the pulse compression. High-intensity few-cycle pulses [770-nm central wavelength, 0.2-mJ pulse energy, 3.5-fs (FWHM) duration] were typically obtained from this system. A part of the uncompressed radiation of this Ti:sapphire laser [pulse energy $120 \mu \mathrm{J}$, pulse duration $8 \mathrm{ps}$ (FWHM), pulse repetition rate $1 \mathrm{kHz}$ ] was split from the beam line prior to the first compressor stage and was focused into the vacuum chamber to create a plasma on an indium target [Fig. 1(a)] placed in a vacuum chamber. The picosecond heating pulses created a plasma plume with a diameter of $\sim 0.5 \mathrm{~mm}$ using an intensity on the target surface of $I_{\mathrm{ps}}=8 \times 10^{9} \mathrm{~W} \mathrm{~cm}^{-2}$. The 3.5 -fs pulses were focused, in a direction orthogonal to that of the heating pulse, into the laser plasma using a $400-\mathrm{mm}$ focal length spherical mirror. The delay between the plasma initiation and the femtosecond pulse propagation, required for formation and expansion of the plasma plume away from the surface of the target, was set to $33 \mathrm{~ns}$. The intensity of this femtosecond driving pulse in the plasma area was estimated to be $I_{\mathrm{fs}}=$ $3 \times 10^{14} \mathrm{~W} \mathrm{~cm}^{-2}$. The generated harmonics were analyzed

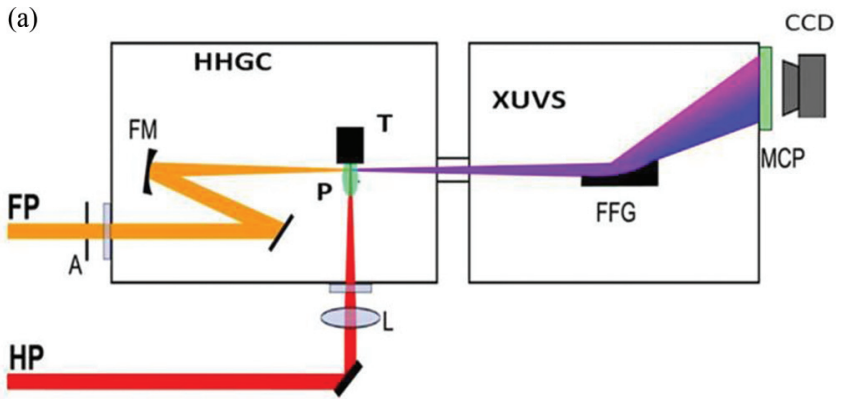

(b)

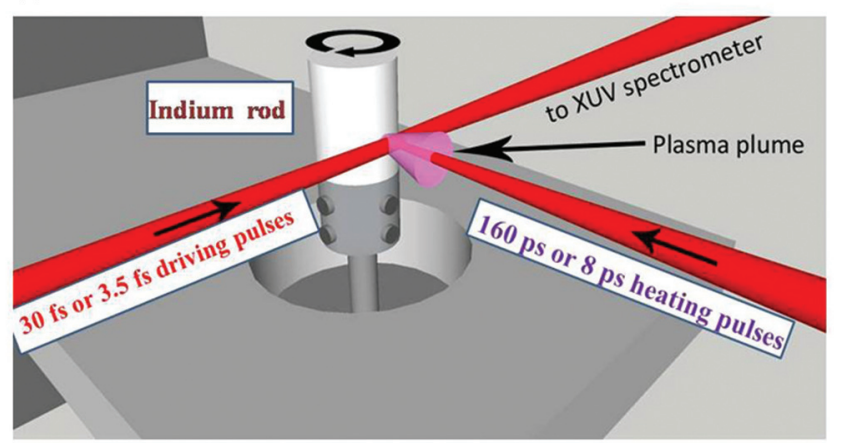

FIG. 1. (Color online) (a) Experimental setup for harmonic generation in plasma plumes. FP, femtosecond harmonic driving pulse (30 fs at FWHM or 3.5 fs at FWHM); HP, picosecond heating pulse (160 ps at FWHM or 8 ps at FWHM); A, aperture; HHGC, high-order-harmonic generation chamber; FM, focusing mirror; L, focusing lens; T, target (rotating In rod); P, plasma plume; XUVS, extreme ultraviolet spectrometer; FFG, flat field grating; MCP, microchannel plate and phosphor screen detector; CCD, CCD camera. (b) Experimental arrangement of rotating target configuration.

by an XUV spectrometer consisting of a flat-field grating (1200 lines $/ \mathrm{mm}$, Hitachi) and a microchannel plate (Photonis USA Inc.) coupled to a phosphor screen. The spatially resolved spectra of the generated harmonics were detected by a CCD camera [29].

We also used longer pulses for HHG in indium plasma plumes using a $1-\mathrm{kHz}$ repetition rate Ti:sapphire laser (Red Dragon, KML Inc.) and the described experimental setup for plasma HHG [Fig. 1(a)]. Similarly, a part of the uncompressed radiation $(780 \mathrm{~nm}, 1.3 \mathrm{~mJ}, 160 \mathrm{ps}$ at FWHM) was split off from the beam line prior to the compressor stage and used for ablation of the indium target. In this case, the resulting intensity on the target surface was $6 \times 10^{9} \mathrm{~W} \mathrm{~cm}^{-2}$. After a delay of $40 \mathrm{~ns}$, the compressed laser pulse $[780 \mathrm{~nm}, 0.6 \mathrm{~mJ}, 30 \mathrm{fs}$ (FWHM)] was focused onto the plasma using a 200-mm focal length spherical mirror up to an intensity of $5 \times 10^{14} \mathrm{~W} \mathrm{~cm}^{-2}$ to generate the high-order harmonics. The position of the focus relative to the plume was chosen to maximize harmonic generation efficiency [30].

The target (an indium rod of diameter of $10 \mathrm{~mm}$ and length of $20 \mathrm{~mm}$ ) was rotated to minimize overheating and damage of its surface from repeated laser shots [Fig. 1(b)], thus ensuring more stable ablation conditions and consequently improving harmonic stability [31]. 


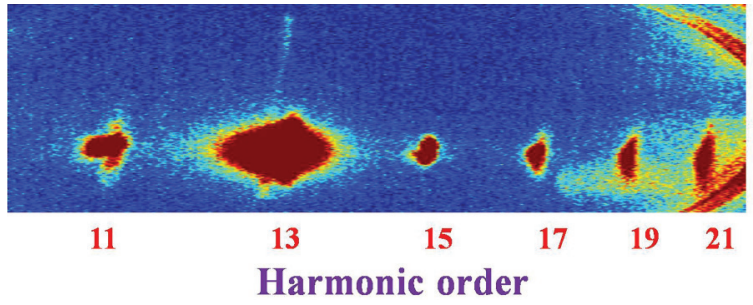

(a)

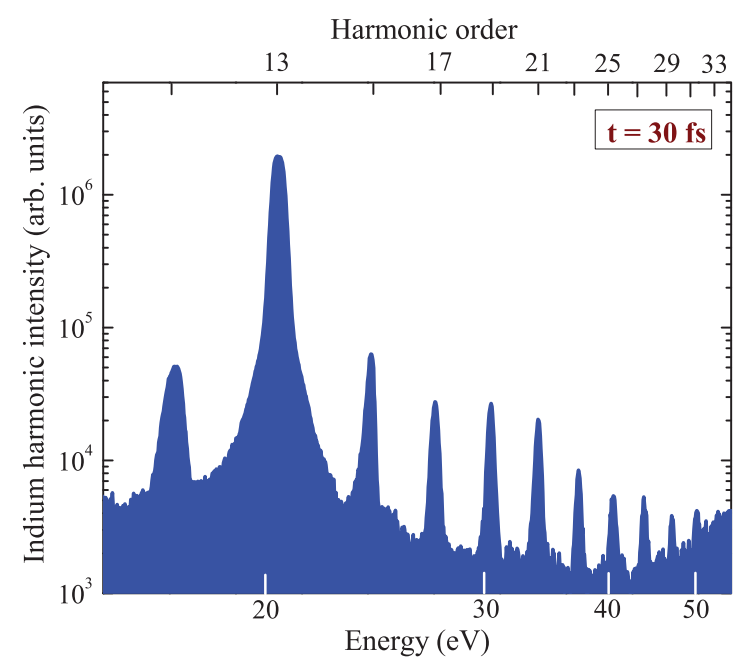

(b)

FIG. 2. (Color online) (a) Raw image of the low-order harmonic spectrum generated from an indium plasma in the case of 30fs (FWHM) driving pulses. The thin line with high divergence above the 13th harmonic is a resonance transition at $19.92 \mathrm{eV}$. (b) Spectral distribution of indium harmonics, along the whole range of harmonic generation, using multicycle (30-fs) pulses. The highest order obtained is the $33 \mathrm{rd}$ harmonic.

\section{INDIUM EMISSION SPECTRA IN THE CASES OF 30-FS AND 3.5-FS DRIVING PULSES}

The harmonic spectrum of indium plasma using 30 -fs pulses showed a strong emission line at $\sim 20.7 \mathrm{eV}(\lambda=$ $60 \mathrm{~nm})$ corresponding to the 13th harmonic of the 780-nm driving radiation (photon energy $E_{\mathrm{p}}=1.59 \mathrm{eV}$ ). This line dominates over the whole spectrum, which also displays weak lines around 17.5 and $23.9 \mathrm{eV}$, corresponding to the 11th and 15 th harmonics, and even weaker higher-order harmonics up to around the 30th orders.

Enhanced 13th harmonic $\left(\lambda=61.5 \mathrm{~nm}, E_{\mathrm{p}} \approx 20.14 \mathrm{eV}\right)$ from In containing plasmas has been reported earlier [21,32-34], using multicycle 10-Hz Ti:sapphire driving lasers operating at $800 \mathrm{~nm}$, and explained as an effect of resonance with the strong In II transition (at $19.92 \mathrm{eV}$ ). In the present experiments, the central wavelength of the 30 -fs driving laser pulses $(780 \mathrm{~nm})$ was shorter than in those previous studies and the photon energy of the 13th harmonic at chirp-free conditions was detuned from the mentioned In II transition.

We were able to optimize the efficiency of the resonant harmonic by tuning it towards the longer wavelength side, so that the frequency of 13th harmonic became closer to the In II resonance line. To this aim, we added a positive chirp to the driving pulse by adjusting the separation of the two gratings in the pulse compressor. Figure 2 shows both a raw image of the low-order range of the HHG spectrum from the indium plasma, after tuning the 13th harmonic wavelength towards the longer wavelength side, and the lineout of harmonic intensities along the whole harmonic spectrum (up to the 33rd order). The ratio between the intensities of the resonantly enhanced harmonic and the neighboring ones exceeded 40 . The dominance of the 13th harmonic in this case becomes even more pronounced as compared with the spectrum obtained before tuning the chirp of driving radiation. One can clearly see the excited resonance line [Fig. 2(a), thin vertical line above the left side of the broad 13th harmonic emission possessing considerably higher divergence than the harmonic emission]. The maximum enhancement of 13th harmonic relative to the neighboring ones was obtained once we approached the resonance transition from the blue side, at a wavelength that did not exactly match the harmonic frequency with that of the ion transition. This peculiarity will be further discussed in Sec. VII.

The spectral pattern of the XUV radiation generated from the In plasma was considerably modified once we changed the driving radiation from multicycle pulses to few-cycle pulses $\left(3.5 \mathrm{fs}, \lambda=770 \mathrm{~nm}, E_{\mathrm{p}}=1.61 \mathrm{eV}\right)$. At a moderate ablation intensity $\left(I_{\mathrm{ps}}=5 \times 10^{9} \mathrm{~W} \mathrm{~cm}^{-2}\right)$, no spontaneous plasma emission (i.e., in absence of excitation with 3.5-fs pulses) was detected in the range of $15-30 \mathrm{eV}$. At these conditions, focusing the few-cycle pulses in the plasma resulted in the appearance of a strong narrow line at $E_{\mathrm{p}} \approx 20 \mathrm{eV}$ [Fig. 3(a)]. No other harmonics and emissions were observed at these conditions, contrary to the case of multicycle pulses. Ablation of the target at higher intensity $\left(I_{\mathrm{ps}}=8 \times 10^{9} \mathrm{~W} \mathrm{~cm}^{-2}\right)$, without the influence of few-cycle driving pulses, also did not result in plasma spontaneous emission. However, in that case the presence of the few-cycle driving pulses led to additional spectral lines mostly in the shorter wavelength side of the strong $\approx 20-\mathrm{eV}$ line [Fig. 3(b)]. One can see the highly divergent In II resonance line at $19.92 \mathrm{eV}$, which displays a maximum intensity in its central part corresponding to small divergence of this radiation. The intensity of the $\approx 20-\mathrm{eV}$ emission exceeded that of other emissions in the shorter wavelength range (at 21.9, 23.3, 24.1, and $25.2 \mathrm{eV}$ ); however, the contrast (i.e., ratio between $20 \mathrm{eV}$ and other emissions intensities) was considerably lower than in the case shown in Fig. 2(b).

None of these relatively narrow spectral lines, at frequencies above $20 \mathrm{eV}$, could be assigned to the plasma harmonics, which should possess much broader bandwidths than those observed lines. The bandwidths of harmonics are defined by that of the driving radiation, which undergoes considerable broadening after the second stage of chirping and compression from $25 \mathrm{fs}$ ( $\sim 60 \mathrm{~nm}$ FWHM) to $3.5 \mathrm{fs}(\sim 215 \mathrm{~nm}$ FWHM) . In the latter case, the positions of the harmonics in the 16- to $30-\mathrm{eV}$ range were defined using a carbon plasma plume, which allowed the generation of featureless 11th to 15th harmonics [Fig. 3(c)] in this spectral range. The central wavelength of the driving radiation $(770 \mathrm{~nm})$ can be retrieved from this spectrum showing the energies of the 11th $\left(E_{\mathrm{p}} \approx 17.7 \mathrm{eV}\right)$, 13 th $\left(E_{\mathrm{p}} \approx 20.9 \mathrm{eV}\right)$, and 15th $\left(E_{\mathrm{p}} \approx 24.15 \mathrm{eV}\right)$ harmonics. The change in the spectral characteristics of the driving radiation led to corresponding changes of harmonic spectra, which were related with the increase of laser intensity, detuning of harmonic wavelength from the ion transitions, and to some 

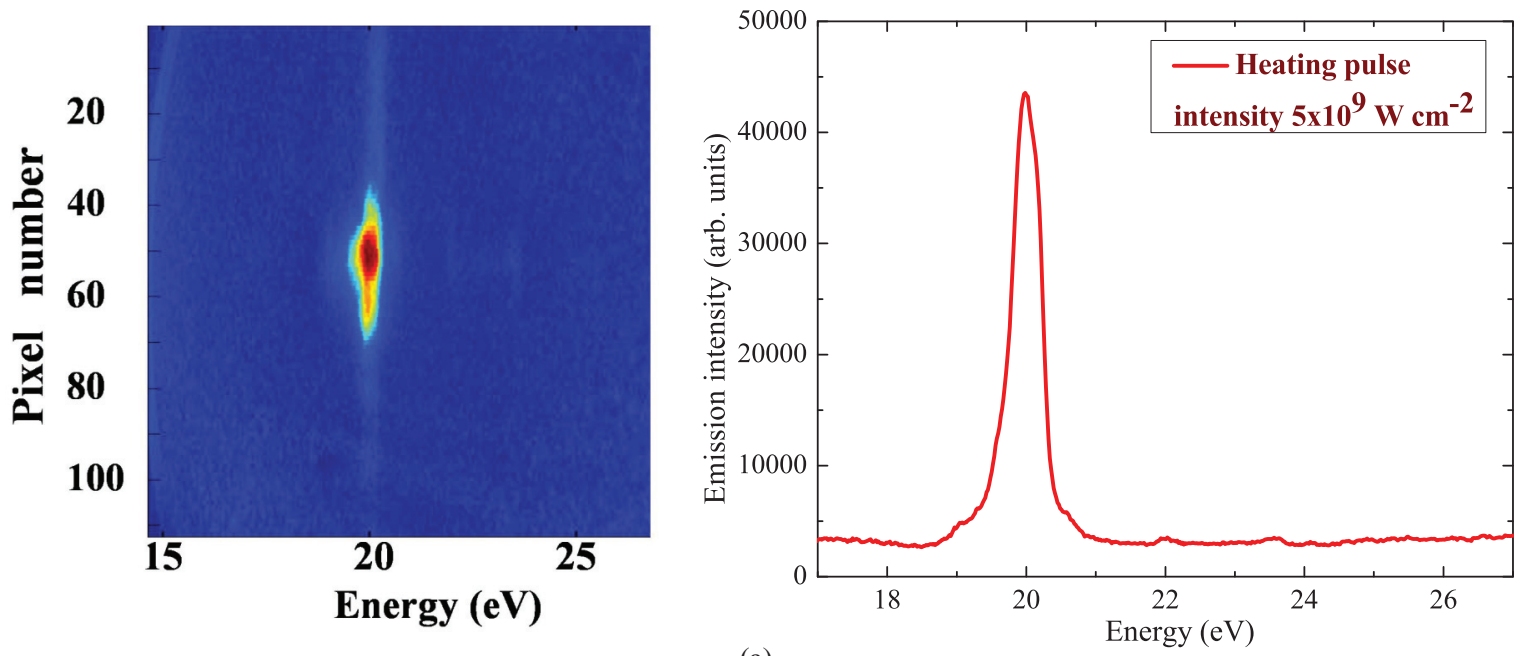

(a)
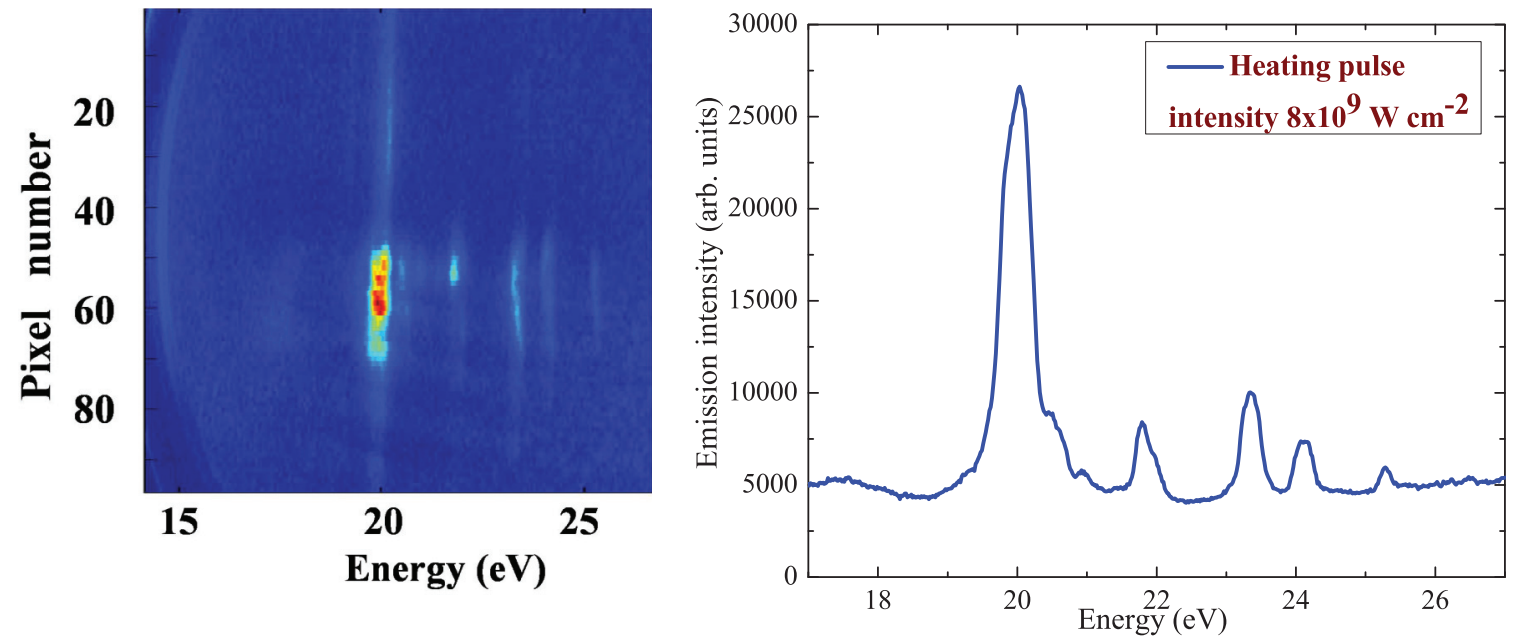

(b)

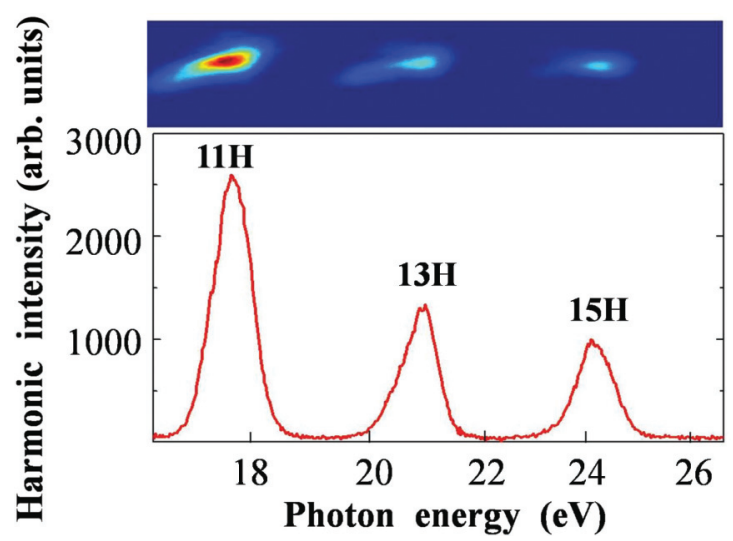

(c)

FIG. 3. (Color online) Raw images and lineouts of the spectra of the indium plasma emission upon excitation by 3.5-fs (FWHM) pulses using the heating pulse intensities of (a) $I_{\mathrm{ps}}=5 \times 10^{9} \mathrm{~W} \mathrm{~cm}^{-2}$ and (b) $I_{\mathrm{ps}}=8 \times 10^{9} \mathrm{~W} \mathrm{~cm}^{-2}$. At low heating pulse intensity (a) one can see the highly divergent 19.92-eV resonance emission and its enhanced central part accompanied with some additional lines in (b). In the latter case the intensity of the 19.92-eV emission exceeds that of other emissions (at $\sim 21.9,23.3,24.1$, and $25.2 \mathrm{eV}$ ), although not as strongly as in the case of emission of the harmonics shown in Figs. 2(b) and 3(a). (c) Raw image and lineout of harmonic spectra from a carbon plasma obtained by excitation with 3.5-fs pulses. Broadband harmonics are observed in this case, which could be distinguished from the emission lines shown in (a) and (b). The 11th, 13th, and 15th harmonic energies are centered at $\sim 17.7 \mathrm{eV}, \sim 20.9 \mathrm{eV}$, and $\sim 24.15 \mathrm{eV}$, respectively. 
other mechanisms, which will be discussed later. Our results show that the strong and relatively narrow $20-\mathrm{eV}$ emission observed in the indium plasma spectrum upon interaction with 3.5-fs pulses does not coincide with either the 11th harmonic or the 13th harmonic of 770-nm broadband driving radiation. In Secs. IV, V, and VII, we discuss the origin of this emission appearing in the case of few-cycle pump and propose a few options for explanation of the features of enhanced low-divergence narrowband $20-\mathrm{eV}$ radiation observed in these studies. Notice that the exact nature of this radiation is still under investigation.

Commonly, for multicycle pulses, the increase of pulse duration can be accomplished by changing the distance between the gratings in the compressor stage. It is a widely used technique, which enables a tuning of gas and plasma harmonic wavelengths from broadband sources (see, for example, $[35,36])$. As it was already mentioned, to tune harmonics towards the longer wavelength side, so that the frequency of the 13th harmonic became closer to the 19.92-eV In II resonance line, we added a positive chirp to the driving pulse by shortening a distance between two gratings in the pulse compressor of the 30-fs, 780-nm laser. Only the wavelength at the leading edge of the pulse contributes significantly to the harmonic generation because, at the intensity used, the strong field generates the multiply ionized plasma that grows increasingly with time, eventually preventing HHG during the trailing part of the pulse (the so-called ionization gating effect).

Another technique for the chirping of laser pulses was introduced in the case of few-cycle pulses. The chirp of these broadband pulses was modified by the fine tuning of the double glass wedges placed after the bunch of chirped mirrors. The change of the path through this wedge pair enabled the variation of spectral distribution along the pulse. Thus the chirp variation in that case was carried out by the movement of the wedges with respect to each other (analogously to the chirp variations in the prism compressors). The description of this technique could be found in [37]. The compressed pulses were characterized with spatially encoded arrangement for spectral shearing interferometry for direct electric field reconstruction (SEA-F-SPIDER) [38]. This technique enabled us to control the pulse duration during modification of the chirp characteristics of a few-cycle pulse.

In the case of few-cycle pulses, we were not able to tune the 13th harmonic wavelength towards the strong In II transition by modifying the laser chirp, as was the case for multicycle pulses. For positively chirped pulses, this is attributed to the largely significant increase of duration for the 3.5-fs pulses and considerable change of experimental conditions for HHG, in contrast with the less significant increase (from 30 to $47 \mathrm{fs}$ ) for multicycle pulses. Therefore, one can assume that only the narrow part of the whole spectrum of the broadband 13th harmonic of the driving chirp-free 770-nm pulses was efficiently converted to the $\approx 20-\mathrm{eV}$ emission (see the discussion of this point in Sec. VII).

\section{TESTING THE INDIUM EMISSION SPECTRA OBTAINED USING 3.5-FS PULSES}

In this section, we address the origin of the enhanced 20-eV emission observed when the 3.5 -fs pulses propagated through the indium plasma. To define the coherent character of this emission we tested the influence of the laser polarization on its intensity. The polarization of the driving radiation was changed by rotating a quarter-wave plate inserted in the driving beam path. With the change of polarization from linear to elliptical, and finally to circular, the intensity of this emission showed a characteristic abrupt decrease, accompanied by the disappearance of shorter wavelength lines. This behavior is a distinctive feature of the harmonic generation mechanism based on the recombination of the returning electron with the parent particle. Notice that in absence of the 3.5-fs excitation pulses, we did not detect spontaneous emissions from the In plasma under optimal ablation conditions.

Another test for the coherent character of this emission was carried out using a Young double-slit interferometer. The double slits were mounted on a translation stage and placed approximately $40 \mathrm{~cm}$ from the targets, with the microchannel plate situated at $70 \mathrm{~cm}$ from the slits. The two slits, spaced by $50 \mu \mathrm{m}$, were made from tungsten and had $6-\mu \mathrm{m}$ width and $10-\mathrm{mm}$ length. The images of interference fringes were taken in a subsequent chamber using a flat field grating, a microchannel plate, and a CCD camera.

Figure 4 shows the typical XUV emission spectrum and the interference fringes obtained after propagation of this radiation through the double-slit interferometer. One can see three enhanced emissions [Fig. 4(a)], which do not coincide with the central wavelengths of the 11th, 13th, and 15th harmonics, although as already mentioned, may represent the enhanced contributions from the parts of those harmonics. The interference patterns of these three lines are shown in Fig. 4(b). The interferometric visibility of these patterns was measured to be $\sim 0.66$.

We did not measure the coherence properties of emission from the indium plasma by overexciting the target using strong heating pulses, without further excitation using 3.5-fs pulses. However, the comparative measurements of spatial coherence at similar conditions were carried out in the case of zinc plasma, which showed a weak visibility of the interference fringes in the case of spontaneous emission of laser-produced plasma, while the visibility of harmonics-induced interference pattern $(\sim 0.72)$ was similar to the case of indium plasma experiments.

The above polarization and interference tests confirmed that the observed enhanced radiation from the indium ablation

(a)

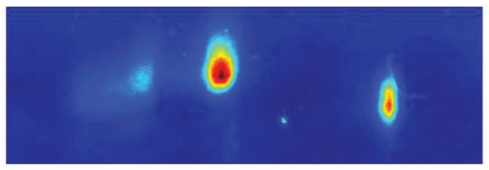

(b)

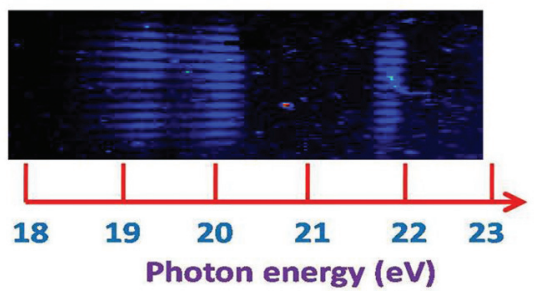

FIG. 4. (Color online) Spectra of emission generated from the In plasma using 3.5-fs pulses (a) without and (b) with double slits. 


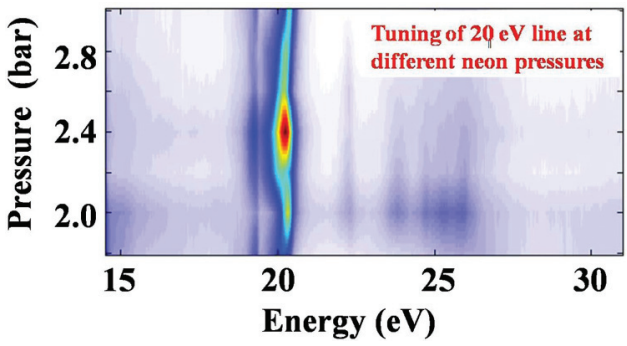

FIG. 5. (Color online) Variation of emission from the indium plasma excited by 3.5 -fs pulses at different pressures of neon in hollow fiber.

plume possesses good coherence and was not originated from plasma spontaneous emission.

The change of the gas backing pressure in the hollow fiber allowed us to vary the laser pulse duration between 3.5 and $25 \mathrm{fs}$. In the present experiments, we varied the $\mathrm{Ne}$ backing pressure in the fiber in order to change the spectrum, pulse duration, and intensity of the ultrashort pulses and subsequently observe the resulting changes in the indium emission spectrum. This is shown in Fig. 5, which indicates that, by increasing the $\mathrm{Ne}$ pressure, the strong $\approx 20-\mathrm{eV}$ emission is slightly tuned and blueshifted. One can see that there is an "optimal" pressure (2.4 bar) at which generation of the $\approx 20-\mathrm{eV}$ emission is achieved with maximum efficiency at other equal experimental parameters.

The blueshift of the $20-\mathrm{eV}$ emission can be explained as follows. The increase of Ne backing pressure leads to a considerable broadening of the laser spectrum, which is followed by the shortening of the pulse duration after compression using the chirped mirrors. The corresponding increase of laser intensity induces a change of the free electron density $(d N / d t)$ and a transient variation of the refractive index, a parameter that strongly depends on the intensity of driving pulses and on the time needed to ionize a considerable fraction of particles. The change in the refractive index induces a shift $\delta \lambda$ of the central laser wavelength $\lambda$ that can be defined as [38]:

$$
\delta \lambda=-\left(e^{2} \lambda^{3} / 2 \pi m_{\mathrm{e}} c^{3}\right) L d N / d t,
$$

where $L$ is the length of medium, $e$ and $m_{\mathrm{e}}$ are the charge and mass of electron, and $c$ is the light velocity. The blueshift of the fundamental radiation $\delta \lambda$ leads to a corresponding blueshift $\delta \lambda_{q}=\delta \lambda / q$ for the $q$ th-order harmonic at $\lambda_{\mathrm{q}}=\lambda / q$. A blueshift of harmonics has been reported previously in gas HHG experiments [40,41], as well as in plasma HHG studies [42]. In [40], the effect of free electrons on the spectral properties of the high-order harmonics was analyzed in a neon gas jet using 30-fs, Ti:sapphire laser pulses. Measurements, carried out at a laser driving intensity of $4 \times 10^{14} \mathrm{~W} \mathrm{~cm}^{-2}$, clearly showed the possibility of continuously tuning the harmonic wavelength by taking advantage of the blueshift induced by the transient appearance of free electrons in the medium.

The "optimization" of the $20-\mathrm{eV}$ emission observed at a pressure of 2.4 bar of neon could be related with the effect induced by tuning the harmonic central wavelength and the creation of better phase-matching conditions for some parts of the harmonic emission spectrum (see the discussion of the possibility of phase matching in the vicinity of resonance presented in Sec. VII). These conditions for the enhancement of the macroscopic propagation effect may appear in some plasma plumes near their ionic or atomic transitions possessing strong oscillation strength. However, the additional influence of the microscopic response cannot be disregarded. This effect is taken into account in the following section.

\section{THEORETICAL CONSIDERATION OF THE MICROSCOPIC RESPONSE}

We solve numerically the three-dimensional timedependent Schrödinger equation (TDSE) for the model system in the linearly polarized laser field (atomic units are used in this section):

$$
i \frac{\partial}{\partial t} \Psi(\vec{r}, t)=\left[-\frac{1}{2} \nabla^{2}+V(r)-E(t) x\right] \Psi(\vec{r}, t),
$$

where $V(r)$ is the parent ion potential, and $x$ is laser field polarization direction. The laser field is given by

$$
E(t)=\left\{\begin{array}{ll}
E_{0}\left(\sin \left(\frac{\pi t}{2 \tau}\right)\right)^{2} \cos \left(\omega_{0} t+\varphi\right), & 0<t<2 \tau \\
0, & t>2 \tau
\end{array},\right.
$$

where $\omega_{0}$ is the fundamental frequency, and $\varphi$ is the pulse CEP. Note that under $\tau$ equal to integer number of optical half cycles (more than one), this field satisfies the following condition: $\int_{-\infty}^{\infty} E(t) d t=0$. This condition implies the absence of a static component of the field and thus should be satisfied for any laser field.

Exact description of an autoionizing state assumes a multielectron dynamics study. However, a numerical TDSE solution for a multielectronic system in an intense laser field is an extremely difficult task (practically possible now only for helium). So we are using a single-active electron approximation, and the role of the other electrons is reproduced with a model potential of the parent ion, as it was done in $[25,28,30]$ :

$$
V(r)=-\frac{2}{\sqrt{a_{0}^{2}+r^{2}}}+a_{1} \exp \left[-\left(\frac{r-a_{2}}{a_{3}}\right)^{2}\right],
$$

where the fitting parameters $a_{0}, a_{1}, a_{2}, a_{3}$ are chosen to reproduce some properties of In II. Namely, as it was mentioned above, the $19.92-\mathrm{eV}$ ion transition $4 d^{10} 5 s^{2}{ }^{1} S_{0} \rightarrow 4 d^{9} 5 s^{2} 5 p$ $\left({ }^{2} D\right){ }^{1} P_{1}$ from the ground state to an AIS has a frequency close to that of the 13th harmonic frequency of the 800 -nm radiation, and an oscillator strength that substantially exceeds those of the neighboring transitions in the spectral region considered [43]. Thus, it is appropriate to neglect other excited states for this ion and to choose the potential parameters $a_{0}=0.65, a_{1}=1.0$, $a_{2}=3.8$, and $a_{3}=1.6$ to reproduce the energies of the ground state and AIS, the AIS width, and the transition oscillator strength.

The numerical method for the TDSE solution is described in [44]. To characterize the XUV field emission we calculated the second derivative of the dipole moment. According to the Ehrenfest's theorem it is equal to the quantum-mechanical expectation value of the force acting on the electron $f(t)=-\left\langle\Psi(\vec{r}, t)\left|\frac{\partial V(r)}{\partial x}\right| \Psi(\vec{r}, t)\right\rangle$. Finally, we find its spectrum by calculating $f(\omega)=\int f(t) \exp (i \omega t) d t$. 


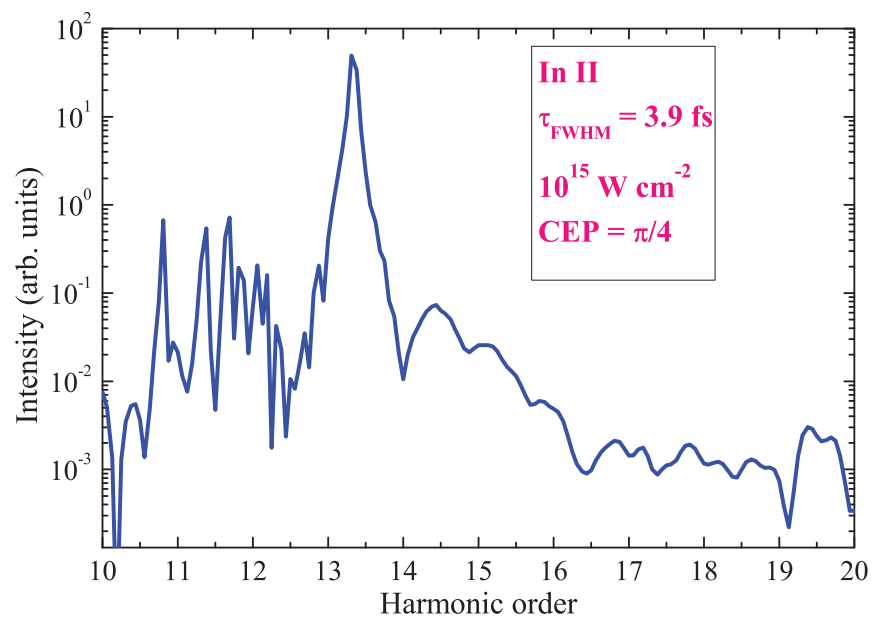

FIG. 6. (Color online) Single-particle response in the XUV range to the 3.9-fs laser pulse calculated via numerical TDSE solution for a model In II ion.

The spectral intensity $[f(\omega)]^{2}$ calculated using $800 \mathrm{~nm}$ laser pulse with the peak intensity of $10^{15} \mathrm{~W} \mathrm{~cm}^{-2}$ and $\tau=4 \pi / \omega$ (corresponding to the pulse duration at half-maximum level of intensity $\tau_{\mathrm{FWHM}}=3.9 \mathrm{fs}$ ) is presented in Fig. 6. Our results show that the enhancement of the XUV generation efficiency near the resonance is even more pronounced, than in the case of a multicycle laser pulse (compare with Fig. 2 from Ref. [25]). In Fig. 7(a) we show the intensity of the XUV emission near the indium resonance (where the intensity was integrated from $12 \omega_{0}$ to $\left.14 \omega_{0}\right)$ as a function of time. One can see that the resonant XUV is emitted mainly in the trailing edge of the laser pulse. Such behavior is very typical, though the details of this dependence are CEP sensitive. This can be attributed to the following two factors. Firstly, the resonant harmonic intensity is proportional to the AIS population, and this population is accumulated during some time, as it was discussed in [25], [28]. Thus, the resonant XUV emission is delayed by this time with respect to the laser pulse envelope, which corresponds

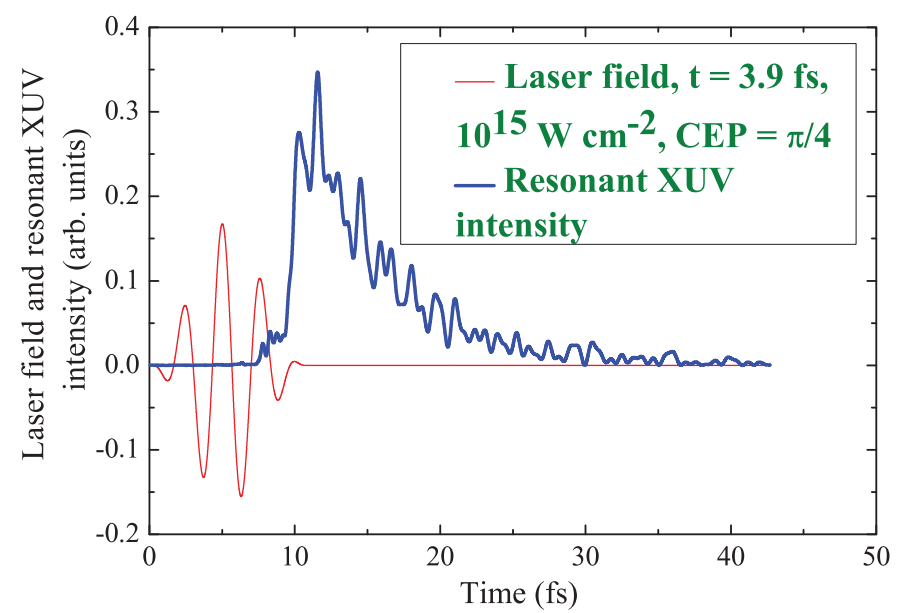

(a) to the AIS lifetime in the field. Although the delay should be less than the field-free resonance lifetime (5.46 fs), due to the photoionization of the AIS, it can be comparable with the laser pulse duration. Secondly, at the trailing edge of the pulse the laser field at the instant of rescattering is less intense, than at the instant of detachment. So the photoionization of the AIS after rescattering is reduced and the XUV emission is more intense. For instance, in conditions of Fig. 7(a) almost all XUV radiation is emitted after the laser pulse has turned off.

We simulated the resonant XUV energy as a function of the laser pulse duration assuming that the pulse energy is constant. The result of this simulation is presented in Fig. 7(b), which shows that there is an optimal laser pulse duration of about 4 fs at which maximum yield could be obtained. This result is comparable with the experimental findings shown in Fig. 5. Thus one can conclude that the experimentally found behavior could be attributed (at least partly) to the properties of the microscopic response.

The resonant XUV energy as a function of the laser pulse CEP is shown in Fig. 8(a). We show this dependence for a given laser intensity, as well the XUV energy averaged over laser intensity (thus taking into account the intensity distribution in the laser beam). One can see that, for the given instant laser intensity, the CEP dependence is significant, but averaging over the laser intensity makes this dependence less pronounced. The resonant XUV energy as a function of the laser intensity is shown in Fig. 8(b). Sharp peaks in this dependence can be attributed to the constructive interference of the contributions to AIS population from different laser half cycles under specific laser intensities.

\section{EXPERIMENTAL STUDIES OF HARMONIC YIELD AT DIFFERENT CEP OF LASER PULSE}

As it was already mentioned, the analysis of single harmonic generation in manganese plasma using 3.5-fs pulses has revealed some interesting features of this process [29].

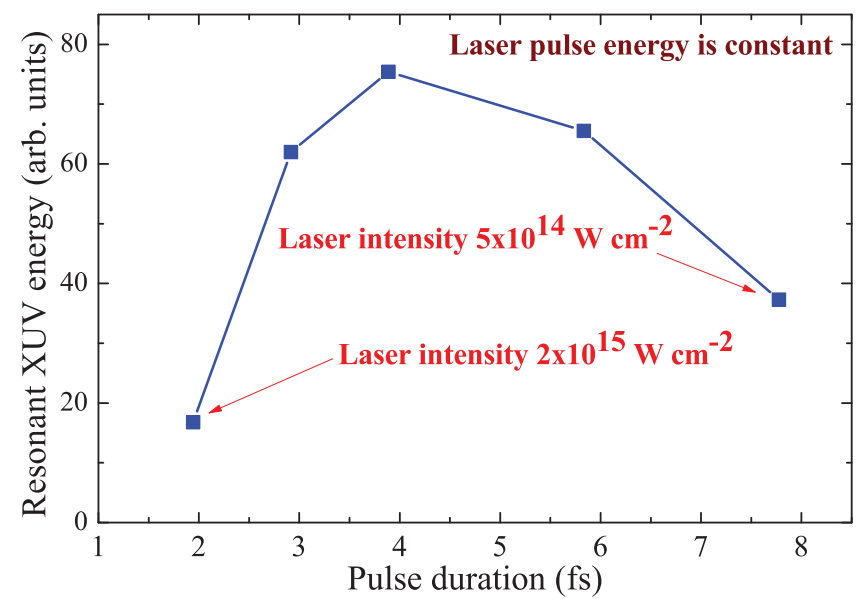

(b)

FIG. 7. (Color online) (a) Intensity of the XUV emission in the vicinity of the ion transition $4 d^{10} 5 s^{2}{ }^{1} S_{0} \rightarrow 4 d^{9} 5 s^{2} 5 p\left({ }^{2} D\right){ }^{1} P_{1}$ from the ground state to an AIS as a function of time. (b) The CEP averaged energy of the resonant XUV emission (from $12 \omega$ to $14 \omega$ ) as a function of the laser pulse duration $\tau_{\mathrm{FWHM}}$ under constant laser pulse energy. 


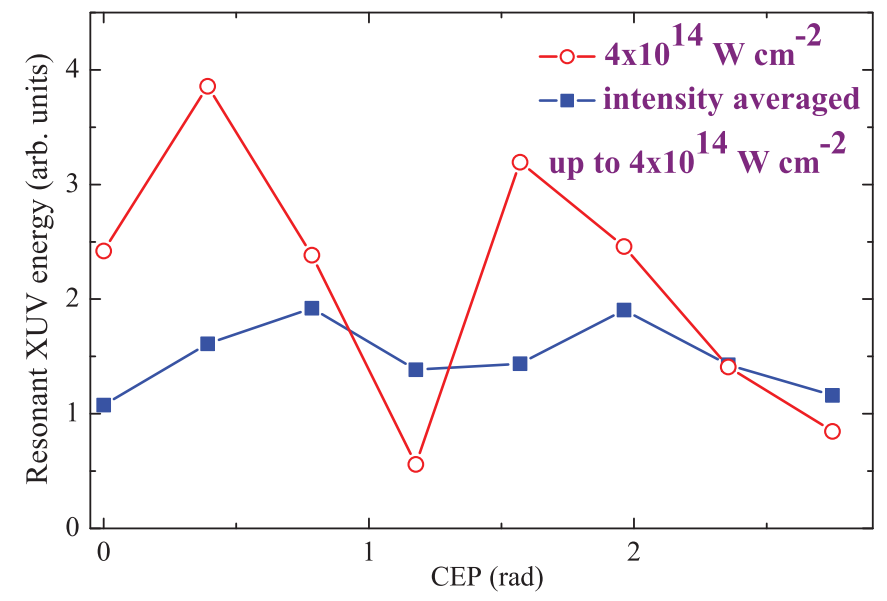

(a)

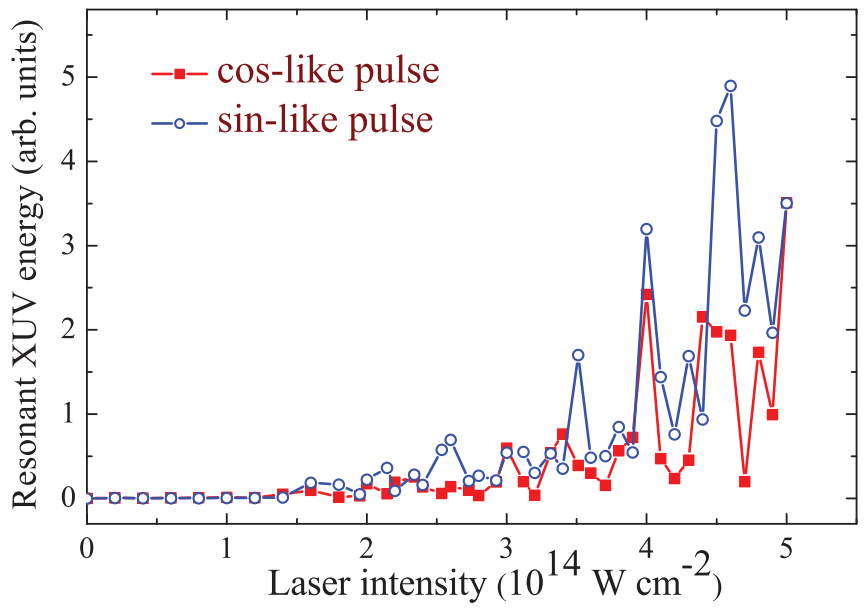

(b)

FIG. 8. (Color online) (a) Resonant XUV energy for the given laser intensity (open circles) and averaged over the laser intensity up to $4 \times 10^{14} \mathrm{~W} \mathrm{~cm}^{-2}$ (solid squares) as a function of CEP of the 3.9-fs pulse. (b) The resonant XUV energy as a function of the peak laser intensity for $\operatorname{CEP} \varphi=0$ (cos-like pulse) and $\operatorname{CEP} \varphi=\pi / 2$ (sin-like pulse).

Particularly, the change of the CEP of few-cycle driving pulse caused only small variations in Mn harmonic spectra. The resonance-enhanced harmonic was generated in the range of 50-51 eV. One can expect that such a pattern of harmonic spectra and weak CEP dependence could be observed as well in the longer wavelength side of XUV spectra. Our studies in In plasma plumes have confirmed this assumption, which can open the doors for development of such coherent, XUV sources and analysis of the spectral properties of emitters through plasma HHG.

Most of the above-described experiments were carried out without CEP stabilization (that is, for random CEP values). To address the theoretical assumptions presented in the previous section, we carried out HHG experiments using the 3.5-fs pulses with stabilized CEP and found some differences in the emission spectra in the case of two phases $(\varphi=0$ and $\pi / 2$; see the normalized spectra of harmonic and plasma emission in Fig. 9). In particular, we observed relatively strong lower-order (11th) harmonic and a broader resonance emission in the case of $\varphi=\pi / 2$. Overall, the spectral shapes of XUV emissions were approximately similar for these two fixed values of CEP.

The experimentally measured harmonic spectra from the indium plasma plumes showed weak dependence on the CEP of driving few-cycle pulses, while the theoretical calculations predicted stronger dependencies. The reason that we did not observe a strong CEP dependence of the harmonic spectra could be attributed, in the case of 3.5-fs pulses, to the presence of the free electrons in the plasma, which might diminish the difference between the HHG spectra recorded for different values of CEP.

The free electrons appearing during propagation of femtosecond pulse through the prepared plasma plume might also influence this process, although the additional concentration of free charges does not considerably increase the initial concentration of electrons. The variation of femtosecond pulse intensity may change the already existing free electron concentration, although we maintained the conditions when no significant amount of free electrons appears during HHG at

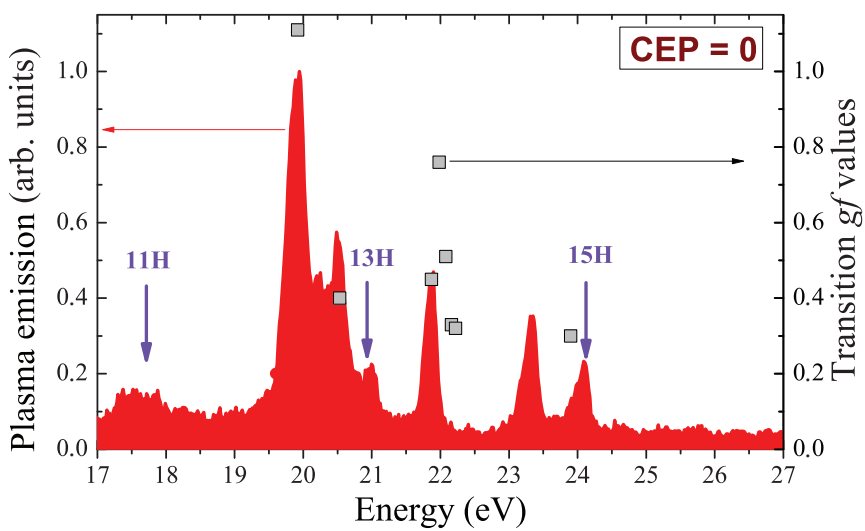

(a)

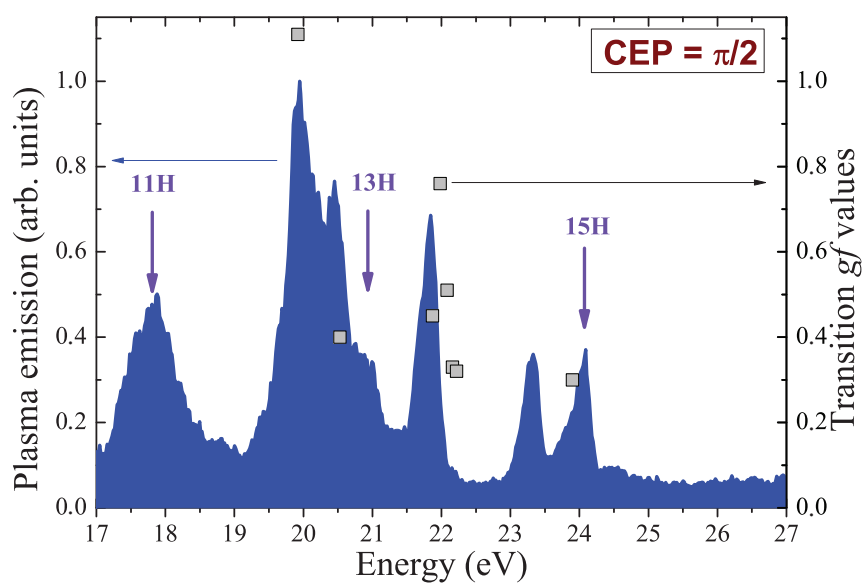

(b)

FIG. 9. (Color online) Harmonic spectra generated from indium plasma in the case of a fixed CEP [(a) $\varphi=0$, (b) $\varphi=\pi / 2$ ] of few-cycle pulses and strong excitation of the indium target. Squares show energies and oscillator strengths ( $g f$ values) of the transitions in In II and In II * taken from Ref. [43] (only transitions with $g f$ values exceeding 0.3 are shown). Arrows indicate the frequencies of the harmonics in carbon plasma shown in Fig. 3(c). 
the intensities of driving femtosecond pulse in the plasma area $\leqslant 3 \times 10^{14} \mathrm{~W} \mathrm{~cm}^{-2}$. Probably, it is possible to analyze the CEP dependence at a variable range of intensities. However, at high intensities, the influence of additionally appeared electrons might decrease the harmonic yield due to the propagation effects induced by the dynamical variation of the dispersion properties of plasma medium. In that case the CEP dependence might be further blurred compared with other effects.

The phase matching during generation of XUV pulses could also blur the CEP dependence of HHG. Indeed, both phase matching and the microscopic harmonic response depend on the ionization degree. If the HHG efficiency in the experiment is limited by inappropriate phase matching, the lower microscopic response for a given CEP (due to lower ionization degree for this CEP) can be compensated for with better phase matching for this CEP (also due to lower ionization degree). In the plasma plume this effect could be more important than for the HHG in gaseous media due to higher ionization. Such behavior of harmonics was confirmed earlier during calculations performed to study the "phase-matching gating" of the generation of an isolated attosecond pulse [45].

One can see in Fig. 9 that almost all spectral peaks can be attributed either to broad harmonics with frequencies close to ones found for carbon plasma, or to transitions in In II and In II * ions described in Ref. [43]. Note that even the spectral peak near $23.3 \mathrm{eV}$ was observed experimentally (see Fig. 1 from [43]), although not attributed to any transition. This agrees with the presentation of the XUV spectrum in the form [46],

$$
\mu(\omega)=F(\omega) \mu_{n r}(\omega),
$$

where $\mu_{n r}(\omega)$ is the spectrum generated in the absence of resonances and the factor $F(\omega)$ describes the enhancement due to the resonance (equal to unity far from a resonance). In the case of a 30-fs laser pulse, $\mu_{n r}(\omega)$ is a comb of narrow harmonics and proximity to a resonance leads to the enhancement of generation of certain harmonic (or harmonics). In the case of a 3.5-fs pulse, the spectrum $\mu_{n r}(\omega)$ is a quasicontinuum, and on top of it the features due to the $F(\omega)$ factor (thus due to resonances) can be seen. Thus the generation of an XUV quasicontinuum with the 3.5 -fs pulses allows the observation of spectral features caused by different transitions in the single spectrum.

\section{DISCUSSION}

We have already pointed out that the enhanced $\approx 20-\mathrm{eV}$ emission did not coincide with either the 11th or the 13th harmonic wavelengths of the 770-nm, 3.5-fs driving laser radiation (as is evident in Fig. 3 in the comparison of the emission spectra of indium and carbon plasmas). For few-cycle driving pulses, plasma emissions at around 22, 23.4, and $24 \mathrm{eV}$ are close to the spectral positions of intense In II $4 d^{10} 5 s 5 p \rightarrow$ $4 d^{9} 5 s 5 p^{2}$ transitions [43]. Since these plasma emission lines do not coincide with the central wavelengths of broadband 11 th, 13th, and 15th harmonics, one can assume that the spectral wings of the harmonics, in resonance with the above ion transitions, became enhanced due to resonance-induced increase of the nonlinear optical response of the plasma. In that case, propagation effects may play a decisive role for efficient phase matching between the driving and harmonic fields in the vicinity of the above-mentioned spectral lines.

Among the factors responsible for the enhancement of individual harmonics, we note the difference between the phase-matching conditions for different harmonics. The phase mismatch varies as the laser pulse propagates through the plasma plume due to further ionization of the nonlinear medium. For harmonics in the plateau region, the phase mismatch attributed to free electrons is one to two orders of magnitude larger than the mismatch due to neutrals and singly charged ions. However, under resonance conditions, when the frequency of a part of a given harmonic becomes close to the frequency of inner-shell atomic transitions, the wave-number variation for this harmonic caused by atoms or ions might be significantly increased and the free electron effect may be canceled. In these circumstances, it is possible to satisfy the optimal phase condition for a part of the single harmonic, with the consequent increase of conversion efficiency for this emission.

The mechanism for improvement of the phase-matching conditions could be as follows. The refractive index of the plasma in the vicinity of a resonant transition $\left(\lambda_{r}\right)$ can be considerably changed thus allowing coincidence of the refractive indices of the plasma at the wavelengths of the driving laser and the part of harmonic emission (Fig. 10). The shortwavelength wing of the resonance showing anomalous dispersion can create the conditions to satisfy this phase-matching condition. One can see that in the area marked by the empty box near $\lambda_{\mathrm{lh}}$, the condition $n_{\mathrm{d}} \approx n_{\mathrm{lh}}$ is fulfilled, where $n_{\mathrm{d}}$ and $n_{\mathrm{lh}}$ are the refractive indices of plasma at the wavelength of driving pulse $\left(\lambda_{d}\right)$ and the long-wavelength side of the harmonic radiation $\left(\lambda_{\mathrm{lh}}\right)$, which central wavelength $\left(\lambda_{\mathrm{ch}}\right)$ stays far from the resonance line.

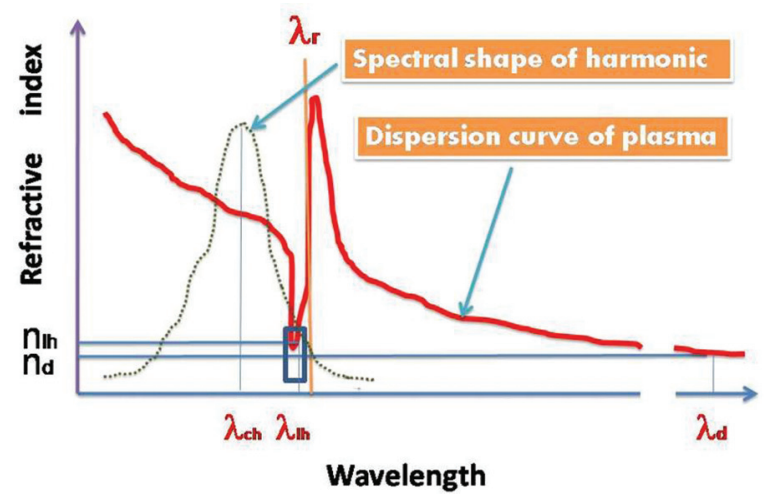

FIG. 10. (Color online) Conditions of the phase matching between the waves of a broadband driving field and a harmonic approaching the short-wavelength side of the resonant transition of the plasma medium. Solid curve shows a dispersion of the refractive index of plasma. Dotted curve represents the spectral shape of a nearby broadband harmonic. The empty box near $\lambda_{\mathrm{lh}}$ shows an enhancement range of the part of harmonic spectrum where the phase mismatch became suppressed. Here $\lambda_{\mathrm{r}}$ is the central wavelength of the ion resonance, $\lambda_{\mathrm{d}}, \lambda_{\mathrm{ch}}$, and $\lambda_{\mathrm{lh}}$ are the wavelengths of the driving radiation, the center of the harmonic, and the long-wavelength side of the harmonic, respectively, and $n_{\mathrm{d}}$ and $n_{\mathrm{lh}}$ are the refractive indices of plasma at $\lambda_{\mathrm{d}}$ and $\lambda_{\mathrm{lh}}$, respectively. 
To analyze this issue in depth one has to know the bandwidths of those resonances, relative role of the nonlinear enhancement of harmonic emission and the absorption properties of plasma in the vicinity of resonances, influence of plasma length on the enhancement factor of the part of harmonic, etc. Currently, there are no data available on these parameters for the indium plasma. Whether this effect affects (or not affects) the phase relations between the interacting waves depends on many factors. Here we just proposed our explanation of the observed enhancement of relatively narrowband emission at the blue side of a strong ionic resonance of indium, which qualitatively coincides with the narrow range of the anomalous dispersion existing at the blue side of this resonance. Figure 10 also explains why we did not obtain the enhanced emission at the exact coincidence of the harmonic and transition wavelengths. This consideration also allows justifying our observation of the enhancement of a narrow part of relatively broad harmonic from the broadband laser source.

Thus, the propagation effects can play a decisive role in optimizing resonance-induced enhancement of harmonics, especially for the broadband driving pulses. The joint influence of the processes at the microscale related with mechanisms described in the four-step model and the macroscopic processes related with phase matching of the interacting waves can create the conditions for the generation of an intense emission possessing the same coherence properties as ordinary harmonics.

Another option to explain the observed In plasma emission lines upon excitation by few-cycle pulses could be related with a lasing effect involving ion transitions. One can assume that, analogously with the x-ray lasers, excitation of low-ionized plasma by ultrashort pulses, increases the population of the discussed excited ion levels, causing stimulated emission at the corresponding wavelengths once population inversion is established between some ion levels. To support this assumption one has to analyze the $I_{\mathrm{e}}(l \times g)$ dependencies, where $I_{\mathrm{e}}$ is the intensity of emitted radiation, $l$ is the length, and $g$ is the gain of the medium, as well as define the saturation conditions of the process. The difficulty in explaining the observed spectral peculiarities of the emission from indium plasma using this approach is related with the unknown values of the lifetimes of the involved excited states and of the ratio between the absorption and the gain of the indium plasma in this spectral range. Moreover, the polarization experiments do not support the lasing assumption, since the gain of the medium should not depend so decisively on the polarization properties of the driving pump laser, as it was observed in our studies.

The important aspect of resonant plasma HHG studies is the temporal characterization of the enhanced harmonic. Measurements of both the femtosecond and attosecond structure of HHG pulses from tin ablation plasma were reported recently in [47]. The 17th harmonic of an $~ 800-n m$ laser was tuned into resonance with the $4 d^{10} 5 s^{2} 5 p^{2} P_{3 / 2} \rightarrow 4 d^{9} 5 s^{2} 5 p^{2}$ $\left({ }^{1} D\right)^{2} D_{5 / 2}$ transition in Sn II. Those studies have shown that the femtosecond envelope of this harmonic does not correspond to a slowly decaying plasma emission, but is indeed slightly shorter than the driving laser pulse. However, the relative phase of the harmonics, which governs the attosecond structure, is significantly perturbed by the resonance. In resonant conditions, the phase locking between the resonant and the neighboring orders is lost, i.e., their relative phase varies significantly within the harmonic spectral width.

At the same time, theoretical modeling of the single enhanced harmonic of sub-4-fs pulse from Mn plasma reported in [29] suggests that this emission could constitute an isolated subfemtosecond pulse. Particularly, the calculations show that the observed 51-eV emission represents subfemtosecond XUV pulses or at least $\sim 1$-fs XUV pulses, for different values of CEP. Those studies have demonstrated the observation of a single, broadband (2.5-eV) 31st harmonic. The spectral bandwidth supported the assumption about the subfemtosecond pulse duration of this radiation, although no temporal measurements were carried out in those studies.

Our present measurements of the bandwidths of the enhanced emission from the indium plasma around $20 \mathrm{eV}$ and above, in the case of excitation by 3.5-fs pulses, did not support the subfemtosecond pulse duration of these XUV pulses. Moreover, the divergence of this radiation exceeds the one reported for a resonant harmonic from a Mn plasma [29]. The possible explanation of the difference in the spatiospectral characteristics in these two cases (i.e., resonance emissions from the indium and manganese plasmas excited by few-cycle pulses) could be related with the different above-described mechanisms involved in the generation of enhanced emission from these two plasma samples.

\section{CONCLUSIONS}

In conclusion, we have analyzed harmonic spectra driven by 30- and 3.5-fs pulses from an indium laser ablation plasma. In both cases the spectra are dominated by an enhanced emission at around $20 \mathrm{eV}$, a distinctive structure that clearly points to the involvement of a resonance centered at this energy. For few-cycle 3.5-fs pulses, the generated XUV spectrum contains several peaks that can be attributed either to wide harmonic lines or to several narrow resonances where the parts of harmonics were enhanced. For multicycle 30-fs pulses, we obtained a plateaulike distribution of harmonics up to the 33rd order, with the 13th harmonic exceeding by around 40 times the adjacent orders. To confirm the nonlinear optical nature of the observed harmonic spectral features originated by few-cycle driving pulses, we analyzed the laser polarization dependence of these emissions and found that strong lines of the indium spectra abruptly disappear with the change of the polarization state from linear to elliptical and further to circular. This provides a clear signature of the emissions being due to high-order-harmonic generation. Coherence measurements also supported our assumption on the nonlinear optical origin of these emission lines.

Our measurements have revealed the influence of the driving pulse duration on the indium plasma emission spectra. The tuning of harmonic energy upon variation of the driving laser pulse duration, controlled by change of gas pressure in the hollow fiber compression system, has shown stabilization of the enhanced energy of the harmonic, an effect that we explain via resonance-induced enhancement at around $20 \mathrm{eV}$. Our theoretical modeling suggests that the emission should depend on the CEP of driving pulse; however, this dependence is essentially reduced after averaging over laser intensity. The experimental observations confirmed a weak influence 
of this parameter on the emission spectra from the indium plasma. The observed weak CEP dependence might reduce the requirements for CEP stabilization of the laser.

\section{ACKNOWLEDGMENTS}

This research was supported by EPSRC programme (Grants No. EP/F034601/1, No. EP/E028063/1, and No. EP/I032517/1) and by European Marie Curie Initial Training Network (Grant No. CA-ITN-214962-FASTQUAST). R.A.G. acknowledges support from the Marie Curie International Incoming Fellowship Grant within the $7^{\text {th }}$ European Community Framework Programme (Grant No. 253104). I.L.-Q. thanks the FPI fellowship (MINECO, Spain) for support, and M.C. thanks Programa Nacional de Movilidad de Recursos Humanos, Plan Nacional de I-D + i 2008-2011, Spain for support. V. V. Strelkov thanks the support from the RFBR (Grants No. 12-02-00627-a and No. 13-02-92623), Ministry of Education and Science of RF (Grant No. MD-6596.2012.2), and the Dynasty Foundation.
[1] P. B. Corkum and F. Krausz, Nat. Phys. 3, 381 (2007).

[2] J. P. Marangos, S. Baker, N. Kajumba, J. S. Robinson, J. W. G. Tisch, and R. Torres, Phys. Chem. Chem. Phys. 10, 35 (2008).

[3] C. Jin, H. J. Wörner, V. Tosa, A.-T. Le, J. B. Bertrand, R. R. Lucchese, P. B. Corkum, D. M. Villeneuve, and C. D. Lin, J. Phys. B: At. Mol. Opt. Phys. 44, 095601 (2011).

[4] U. Fano, Phys. Rev. 124, 1866 (1961).

[5] F. Keller and H. Lefebvre-Brion, Z. Phys. D: At. Mol. Clusters 4, 15 (1986).

[6] M. Y. Amusia and J.-P. Connerade, Rep. Prog. Phys. 63, 41 (2000).

[7] K. J. Schafer, B. Yang, L. F. DiMauro, and K. C. Kulander, Phys. Rev. Lett. 70, 1599 (1993).

[8] P. B. Corkum, Phys. Rev. Lett. 71, 1994 (1993).

[9] M. Lewenstein, P. Balcou, M. Y. Ivanov et al., Phys. Rev. A 49, 2117 (1994).

[10] J. Levesque, D. Zeidler, J. P. Marangos, P. B. Corkum, and D. M. Villeneuve, Phys. Rev. Lett. 98, 183903 (2007).

[11] R. A. Ganeev, L. B. Elouga Bom, J.-C. Kieffer, and T. Ozaki, Phys. Rev. A 76, 023831 (2007).

[12] A. D. Shiner, B. E. Schmidt, C. Trallero-Herrero, H. J. Wörner, S. Patchkovskii, P. B. Corkum, J.-C. Kieffer, F. Légaré, and D. M. Villeneuve, Nat. Phys. 7, 464 (2011).

[13] J. F. Reintjes, Nonlinear Optical Parametric Processes in Liquids and Gases (Academic Press, New York, 1984).

[14] L. Plaja and L. Roso, J. Modern Opt. 40, 793 (1993).

[15] E. S. Toma, P. Antoine, A. de Bohan, and H. G. Muller, J. Phys. B: At. Mol. Opt. Phys. 32, 5843 (1999).

[16] M. B. Gaarde and K. J. Schafer, Phys. Rev. A 64, 013820 (2001).

[17] Z. Zeng, R. Li, Y. Cheng, W. Yu, and Z. Xu, Phys. Scripta 66, 321 (2002).

[18] C. Figueira de Morisson Faria, R. Kopold, W. Becker, and J. M. Rost, Phys. Rev. A 65, 023404 (2002).

[19] R. Taieb, V. Veniard, J. Wassaf, and A. Maquet, Phys. Rev. A 68, 033403 (2003).

[20] P. Ackermann, H. Münch, and T. Halfmann, Opt. Express 20, 13824 (2012).

[21] R. A. Ganeev, M. Suzuki, T. Ozaki, M. Baba, and H. Kuroda, Opt. Lett. 31, 1699 (2006).

[22] R. A. Ganeev, High-Order Harmonic Generation in Laser Plasma Plumes (Imperial College Press, London, 2012).

[23] I. A. Kulagin and T. Usmanov, Opt. Lett. 34, 2616 (2009).

[24] P. V. Redkin and R. A. Ganeev, Phys. Rev. A 81, 063825 (2010).

[25] V. Strelkov, Phys. Rev. Lett. 104, 123901 (2010).

[26] D. B. Milošević, Phys. Rev. A 81, 023802 (2010).

[27] M. V. Frolov, N. L. Manakov, and A. F. Starace, Phys. Rev. A 82, 023424 (2010).
[28] M. Tudorovskaya and M. Lein, Phys. Rev. A 84, 013430 (2011).

[29] R. A. Ganeev, T. Witting, C. Hutchison, F. Frank, M. Tudorovskaya, M. Lein, W. A. Okell, A. Zaïr, J. P. Marangos, and J. W. G. Tisch, Opt. Express 20, 25239 (2012).

[30] R. A. Ganeev, V. V. Strelkov, C. Hutchison, A. Zaï, D. Kilbane, M. A. Khokhlova, and J. P. Marangos, Phys. Rev. A 85, 023832 (2012).

[31] C. Hutchison, R. A. Ganeev, T. Witting, F. Frank, W. A. Okell, J. W. G. Tisch, and J. P. Marangos, Opt. Lett. 37, 2064 (2012).

[32] R. A. Ganeev, Open Spectrosc. J. 3, 1 (2009).

[33] R. A. Ganeev, H. Singhal, P. A. Naik, V. Arora, U. Chakravarty, J. A. Chakera, R. A. Khan, I. A. Kulagin, P. V. Redkin, M. Raghuramaiah, and P. D. Gupta, Phys. Rev. A 74, 063824 (2006).

[34] R. A. Ganeev, L. B. Elouga Bom, J.-C. Kieffer, and T. Ozaki, Phys. Rev. A 75, 063806 (2007).

[35] H. T. Kim, J. H. Kim, D. G. Lee, K. H. Hong, Y. S. Lee, V. Tosa, and C. H. Nam, Phys. Rev. A 69, 031805 (2004).

[36] V. Tosa, H. T. Kim, I. J. Kim, and C. H. Nam, Phys. Rev. A 71, 063808 (2005).

[37] J. S. Robinson, C. A. Haworth, H. Teng, R. A. Smith, J. P. Marangos, and J. W. G. Tisch, Appl. Phys. B 85, 525 (2006).

[38] T. Witting, F. Frank, C. A. Arrell, W. A. Okell, J. P. Marangos, and J. W. G. Tisch, Opt. Lett. 36, 1680 (2011).

[39] E. Yablonovitch, Phys. Rev. Lett. 60, 795 (1988).

[40] C. Altucci, R. Bruzzese, C. de Lisio, M. Nisoli, S. Stagira, S. De Silvestri, O. Svelto, A. Boscolo, P. Ceccherini, L. Poletto, G. Tondello, and P. Villoresi, Phys. Rev. A 61, 021801(R) (2000).

[41] C. A. Froud, E. T. F. Rogers, D. C. Hanna, W. S. Brocklesby, M. Praeger, A. M. de Paula, J. J. Baumberg, and J. G. Frey, Opt. Lett. 31, 374 (2006).

[42] R. A. Ganeev, C. Hutchison, T. Witting, F. Frank, W. A. Okell, A. Zaï, S. Weber, P. V. Redkin, D. Y. Lei, T. Roschuk, S. A. Maier, I. López-Quintás, M. Martín, M. Castillejo, J. W. G. Tisch, and J. P. Marangos, J. Phys. B: At. Mol. At. Phys. 45, 165402 (2012).

[43] D. Duffy and P. Dunne, J. Phys. B: At. Mol. Opt. Phys. 34, L173 (2001).

[44] V. V. Strelkov, A. F. Sterjantov, N. Y. Shubin, and V. T. Platonenko, J. Phys. B: At. Mol. Opt. Phys. 39, 577 (2006).

[45] V. V. Strelkov, E. Mével, and E. Constant, New J. Phys. 10, 083040 (2008).

[46] V. V. Strelkov, M. A. Khokhlova, and N. Yu. Shubin, arXiv:1307.5241.

[47] S. Haessler, V. Strelkov, L. B. Elouga Bom, M. Khokhlova, O. Gobert, J.-F. Hergott, F. Lepetit, M. Perdrix, T. Ozaki, and P. Salières, New J. Phys. 15, 013051 (2013). 